\title{
Solutions of perturbed $p$-Laplacian equation with critical nonlinearity and magnetic fields
}

Huixing Zhang* and Juan Jiang

\section{"Correspondence:}

huixingzhangcumt@163.com Department of Mathematics, China University of Mining and

Technology, Xuzhou, Jiangsu 221116, People's Republic of China

\begin{abstract}
In this paper, we consider a perturbed $p$-Laplacian equation with critical nonlinearity and magnetic fields on $\mathbb{R}^{N}$. By using the variational method, we establish the existence of nontrivial solutions of the least energy.

MSC: 35B33; 35J60; 35J65

Keywords: $p$-Laplacian equation; critical nonlinearity; magnetic fields; mountain pass theorem
\end{abstract}

\section{Introduction}

In this paper, we are concerned with the existence of nontrivial solutions to the following perturbed $p$-Laplacian equation with critical nonlinearity and magnetic fields of the form

$$
-\varepsilon^{p} \Delta_{p, A} u+V(x)|u|^{p-2} u=K(x)|u|^{p^{*}-2} u+f\left(x,|u|^{p}\right)|u|^{p-2} u, \quad x \in \mathbb{R}^{N},
$$

where $\Delta_{p, A} u=\operatorname{div}\left(|\nabla u+i A(x) u|^{p-2}(\nabla u+i A(x) u)\right), i$ is the imaginary unit, $A(x): \mathbb{R}^{N} \rightarrow \mathbb{R}^{N}$ is a real vector potential, $1<p<N, p^{*}=N p /(N-p)$ denotes the Sobolev critical exponent and $N \geq 3$.

This paper is motivated by some works concerning the nonlinear Schrödinger equation with magnetic fields of the form

$$
\begin{aligned}
i \hbar \frac{\partial \psi}{\partial t}= & -\frac{\hbar^{2}}{2 m}(\nabla+i A(x))^{2} \psi+W(x) \psi \\
& -K(x)|\psi|^{2^{*}-2} \psi-h\left(x,|\psi|^{2}\right) \psi \quad \text { for } x \in \mathbb{R}^{N},
\end{aligned}
$$

where $\hbar$ is Planck's constant, $i$ is the imaginary unit, $2^{*}=\frac{2 N}{N-2}(N \geq 3)$ is the critical exponent, $A(x): \mathbb{R}^{N} \rightarrow \mathbb{R}^{N}$ is a real vector potential, $B=\operatorname{curl} A$ and $W(x)$ is a scalar electric potential.

In physics, we are interested in the standing wave solutions, that is, solutions to (1.2) of the type

$$
\psi(x, t)=\exp \left(-\frac{i E t}{\hbar}\right) u(x)
$$

O2013 Zhang and Jiang; licensee Springer. This is an Open Access article distributed under the terms of the Creative Commons Attribution License (http://creativecommons.org/licenses/by/2.0), which permits unrestricted use, distribution, and reproduction in any medium, provided the original work is properly cited. 
where $\hbar$ is a sufficiently small constant, $E$ is a real number, and $u(x)$ is a complex-valued function satisfying

$$
-(\nabla+i A(x))^{2} u(x)+\lambda(W(x)-E) u(x)=\lambda K(x)|u|^{2^{*}-2} u+\lambda h\left(x,|u|^{2}\right) u, \quad x \in \mathbb{R}^{N} .
$$

We can conduct the transition from quantum mechanics to classical mechanics by letting $\hbar \rightarrow 0$. Thus, the existence of semiclassical solutions has a great charm in physical interest.

Problem (1.3) with $A(x) \equiv 0$ has an extensive literature. Different approaches have been taken to investigate this problem under various hypotheses on the potential and nonlinearity. See for example [1-18] and the references therein. The above-mentioned papers mostly concentrated on the nonlinearities with subcritical conditions. Floer and Weinstein in [11] first studied the existence of single and multiple spike solutions based on the Lyapunov-Schmidt reductions. Subsequently, Oh $[16,17]$ extended the results in a higher dimension. Kang and Wei [14] established the existence of positive solutions with any prescribed number of spikes, clustering around a given local maximum point of the potential function. In accordance with the Sobolev critical nonlinearities, there have been many papers devoted to studying the existence of solutions to elliptic boundary-valued problems on bounded domains after the pioneering work by Brézis and Nirenberg [4]. Ding and Lin [8] first studied the existence of semi-classical solutions to the problem on the whole space with critical nonlinearities and established the existence of positive solutions, as well as of those that change sign exactly once. They also obtained multiplicity of solutions when the nonlinearity is odd.

As far as problem (1.3) in the case of $A(x) \neq 0$ is concerned, we recall Bartsch [2], Cingolani [5] and Esteban and Lions [10]. This kind of paper first appeared in [10]. The authors obtained the existence results of standing wave solutions for fixed $\hbar>0$ and special classes of magnetic fields. Cingolani [5] proved that the magnetic potential $A(x)$ only contributes to the phase factor of the solitary solutions for $\hbar>0$ sufficiently small. For more results, we refer the reader to [19-21] and the references therein.

For general $p \geq 1$, most of the works studied the existence results to equation (1.1) with $A(x) \equiv 0$. See, for example, [22-28] and the references therein. These papers are mostly devoted to the study of the existence of solutions to the problem on bounded domains with the Sobolev subcritical nonlinearities.

However, to our best knowledge, it seems that there is no work on the existence of semiclassical solutions to perturbed $p$-Laplacian equation on $\mathbb{R}^{N}$ involving critical nonlinearity and magnetic fields. In this paper, we consider problem (1.1) with magnetic fields. The main difficulty in the case is the lack of compactness of the energy functional associated to equation (1.1) because of unbounded domain $\mathbb{R}^{N}$ and critical nonlinearity. At the same time, we must consider complex-valued functions for the appearance of electromagnetic potential $A(x)$. To overcome this difficulty, we chiefly follow the ideas of [5]. Notice that although the ideas were used in other problems, the adaption of the procedure to our problem is not trivial at all. We need to make careful and complex estimates and prove that the energy functional possesses a Palais-Smale sequence, which has a strongly convergent sequence.

We make the following assumptions on $V(x), A(x), f(x, s)$ and $K(x)$ throughout the paper: 
$\left(\mathrm{V}_{0}\right) \quad V \in C\left(\mathbb{R}^{N}\right), V(0)=\inf _{x \in \mathbb{R}^{N}} V(x)=0$, and there exists $b>0$ such that the set $v^{b}:=\{x \in$ $\left.\mathbb{R}^{N}: V(x)<b\right\}$ has a finite Lebesgue measure;

$\left(\mathrm{A}_{0}\right) \quad A \in C\left(\mathbb{R}^{N}, \mathbb{R}^{N}\right)$ and $A(0)=0$;

$\left(\mathrm{K}_{0}\right) K(x) \in C\left(\mathbb{R}^{N}, \mathbb{R}^{+}\right), 0<\inf K \leq \sup K<\infty$;

$\left(\mathrm{H}_{1}\right) f \in C\left(\mathbb{R}^{N} \times \mathbb{R}^{+}, \mathbb{R}\right)$ and $f(x, s)=o(|s|)$ uniformly in $x$ as $s \rightarrow 0$;

$\left(\mathrm{H}_{2}\right)$ there are $c_{1}>0$ and $p<\alpha<p^{*}$ such that $|f(x, s)| \leq c_{1}\left(1+|s|^{\frac{\alpha-p}{p}}\right)$ for all $(x, s)$;

$\left(\mathrm{H}_{3}\right)$ there exist $a_{0}>0, q>p$ and $\theta \in\left(p, p^{*}\right)$ such that $F(x, s) \geq p a_{0}|s|^{\frac{q}{p}}$ and $\frac{\theta}{p} F(x, s) \leq$ $f(x, s) s$ for all $(x, s)$, where $F(x, s)=\int_{0}^{s} f(x, t) d t$.

Our main result is the following.

Theorem 1 Assume that $\left(\mathrm{V}_{0}\right),\left(\mathrm{A}_{0}\right),\left(\mathrm{K}_{0}\right)$ and $\left(\mathrm{H}_{1}\right)-\left(\mathrm{H}_{3}\right)$ hold. Then for any $\sigma>0$, there exists $\varepsilon_{\sigma}>0$ such that if $\varepsilon \leq \varepsilon_{\sigma}$, equation (1.1) has at least one positive least energy solution $u_{\varepsilon}$, which satisfies

$$
\frac{\theta-p}{p \theta} \int_{\mathbb{R}^{N}}\left(\varepsilon^{p}\left|\nabla u_{\varepsilon}\right|^{p}+V(x)\left|u_{\varepsilon}\right|^{p}\right) \leq \sigma \varepsilon^{N} .
$$

The paper is organized as follows. In Section 2, we give some necessary preliminaries. Section 3 is devoted to the technical lemmas. The proof of Theorem 2 is given in the last section.

\section{Preliminaries}

Let $\lambda=\varepsilon^{-p}$. Equation (1.1) reads then as

$$
-\Delta_{p, A} u+\lambda V(x)|u|^{p-2} u=\lambda K(x)|u|^{p^{*}-2} u+\lambda f\left(x,|u|^{p}\right)|u|^{p-2} u, \quad x \in \mathbb{R}^{N} .
$$

We are going to prove the following result.

Theorem 2 Assume that $\left(\mathrm{V}_{0}\right),\left(\mathrm{A}_{0}\right),\left(\mathrm{K}_{0}\right)$ and $\left(\mathrm{H}_{1}\right)-\left(\mathrm{H}_{3}\right)$ are satisfied. Then for any $\sigma>0$, there exists $\lambda_{\sigma}>0$ such that if $\lambda>\lambda_{\sigma}$, then equation (2.1) has at least one solution of least energy $u_{\lambda}$ satisfying

$$
\frac{\theta-p}{p \theta} \int_{\mathbb{R}^{N}}\left(\left|\nabla u_{\lambda}\right|^{p}+\lambda V(x)\left|u_{\lambda}\right|^{p}\right) \leq \sigma \lambda^{1-\frac{N}{p}} .
$$

In order to prove these theorems, we introduce the space

$$
E_{\lambda, A}=\left\{u \in W^{1, p}\left(\mathbb{R}^{N}, \mathbb{C}\right): \int_{\mathbb{R}^{N}} \lambda V(x)|u|^{p}<\infty, \lambda>0\right\}
$$

equipped with the norm

$$
\|u\|_{\lambda, A}=\left(\int_{\mathbb{R}^{N}}\left(\left|\nabla u+i \lambda^{\frac{1}{p}} A(x) u\right|^{p}+\lambda V(x)|u|^{p}\right)\right)^{\frac{1}{p}} .
$$

It is known that $E_{\lambda, A}$ is the closure of $C_{0}^{\infty}\left(\mathbb{R}^{N}, \mathbb{C}\right)$. Similar to the diamagnetic inequality [10], we have the following inequality

$$
|\nabla| u(x)|| \leq\left|\nabla u+i \lambda^{\frac{1}{p}} A u\right| .
$$


In fact, since $A(x)$ is real-valued, one has

$$
|\nabla| u(x)|| \leq\left|\nabla u \frac{\bar{u}}{|u|}\right|=\left|\operatorname{Re}\left(\nabla u+i \lambda^{\frac{1}{p}} A u\right) \frac{\bar{u}}{|u|}\right| \leq\left|\nabla u+i \lambda^{\frac{1}{p}} A u\right|
$$

(the bar denotes a complex conjugation). This inequality implies that if $u \in E_{\lambda, A}$, then $|u| \in$ $W^{1, p}\left(\mathbb{R}^{N}\right)$, and, therefore, $u \in L^{q}\left(\mathbb{R}^{N}\right)$ for any $q \in\left[p, p^{*}\right)$. That is, if $u_{n} \rightarrow u$ in $E_{\lambda, A}$, then $u_{n} \rightarrow u$ in $L_{\text {loc }}^{q}\left(\mathbb{R}^{N}\right)$ for any $q \in\left[p, p^{*}\right)$ and $u_{n} \rightarrow u$ a.e. in $\mathbb{R}^{N}$.

Solutions of (2.1) will be sought in the Sobolev space $E_{\lambda, A}$ as critical points of the functional

$$
\begin{aligned}
I_{\lambda}(u) & =\frac{1}{p} \int_{\mathbb{R}^{N}}\left(\left|\nabla u+i \lambda^{\frac{1}{p}} A u\right|^{p}+\lambda V(x)|u|^{p}\right)-\frac{\lambda}{p^{*}} \int_{\mathbb{R}^{N}} K(x)|u|^{p^{*}}-\frac{\lambda}{p} \int_{\mathbb{R}^{N}} F\left(x,|u|^{p}\right) \\
& =\frac{1}{p}\|u\|_{\lambda, A}^{p}-\lambda \int_{\mathbb{R}^{N}} G(x, u),
\end{aligned}
$$

where $G(x, u)=\frac{1}{p^{*}} K(x)|u|^{p^{*}}+\frac{1}{p} F\left(x,|u|^{p}\right)$.

It is easy to see that $I_{\lambda}$ is a $C^{1}$-functional on $E_{\lambda, A}[29]$.

\section{Behavior of $(P S)_{c}$ sequence and a mountain pass structure}

In this section, we commence by establishing the necessary results which complete the proof of Theorem 2 .

Lemma 3.1 Let $\left(\mathrm{V}_{0}\right),\left(\mathrm{A}_{0}\right),\left(\mathrm{K}_{0}\right)$ and $\left(\mathrm{H}_{1}\right)-\left(\mathrm{H}_{3}\right)$ be satisfied. For the $(P S)_{c}$ sequence $\left\{u_{n}\right\} \subset$ $E_{\lambda, A}$ for $I_{\lambda}$, we get that $c \geq 0$ and $\left\{u_{n}\right\}$ is bounded in the space $E_{\lambda, A}$.

Proof Under assumptions $\left(\mathrm{K}_{0}\right)$ and $\left(\mathrm{H}_{3}\right)$, we have

$$
\begin{aligned}
& I_{\lambda}\left(u_{n}\right)-\frac{1}{\theta} I_{\lambda}^{\prime}\left(u_{n}\right) u_{n} \\
& =\left(\frac{1}{p}-\frac{1}{\theta}\right)\left\|u_{n}\right\|_{\lambda, A}^{p}+\left(\frac{1}{\theta}-\frac{1}{p^{*}}\right) \lambda \int_{\mathbb{R}^{N}} K(x)\left|u_{n}\right|^{p^{*}} \\
& \quad+\lambda \int_{\mathbb{R}^{N}}\left(\frac{1}{\theta} f\left(x,\left|u_{n}\right|^{p}\right)\left|u_{n}\right|^{p}-\frac{1}{p} F\left(x,\left|u_{n}\right|^{p}\right)\right) .
\end{aligned}
$$

In connection with the facts that $I_{\lambda}\left(u_{n}\right) \rightarrow c$ and $I_{\lambda}^{\prime}\left(u_{n}\right) \rightarrow 0$ as $n \rightarrow \infty$, we obtain that the $(P S)_{c}$ sequence $\left\{u_{n}\right\}$ is bounded in $E_{\lambda, A}$, and the energy level $c \geq 0$.

Next, let $\left\{u_{n}\right\}$ denote a $(P S)_{c}$ sequence. By Lemma 3.1, it is bounded, thus, without loss of generality, we may assume that $u_{n} \rightarrow u$ in $E_{\lambda, A}$. Furthermore, passing to a subsequence, we have $u_{n} \rightarrow u$ in $L_{\mathrm{loc}}^{q}\left(\mathbb{R}^{N}\right)$ for any $q \in\left[p, p^{*}\right)$ and $u_{n} \rightarrow u$ a.e. in $\mathbb{R}^{N}$.

Lemma 3.2 For any $s \in\left[p, p^{*}\right)$, there is a subsequence $\left\{u_{n_{j}}\right\}$ such that for any $\varepsilon>0$, there exists $r_{\varepsilon}>0$ with

$$
\lim _{j \rightarrow \infty} \sup \int_{B_{j} \backslash B_{r}}\left|u_{n_{j}}\right|^{s} \leq \varepsilon \quad \text { for any } r \geq r_{\varepsilon}
$$

where $B_{r}:=\left\{x \in \mathbb{R}^{N}:|x| \leq r\right\}$. 
Proof It is easily obtained by the similar proof of Lemma 3.2 [8].

Let $\eta \in C^{\infty}\left(\mathbb{R}^{+}\right)$be a smooth function satisfying $0 \leq \eta(t) \leq 1, \eta(t)=1$ if $t \leq 1$ and $\eta(t)=0$ if $t \geq 2$. Define $\widetilde{u}_{j}(x)=\eta(2|x| / j) u(x)$. It is not difficult to see that

$$
\left\|u-\tilde{u}_{j}\right\|_{\lambda, A} \rightarrow 0 \quad \text { as } j \rightarrow \infty
$$

Lemma 3.3 One has

$$
\begin{aligned}
& \lim _{j \rightarrow \infty} \sup \operatorname{Re} \int_{\mathbb{R}^{N}}\left(f\left(x,\left|u_{n_{j}}\right|^{p}\right)\left|u_{n_{j}}\right|^{p-2} u_{n_{j}}\right. \\
& \left.\quad-f\left(x, u_{n_{j}}-\widetilde{u}_{j}\right)\left|u_{n_{j}}-\widetilde{u}_{j}\right|^{p-2}\left(u_{n_{j}}-\widetilde{u}_{j}\right)-f\left(x,\left|\widetilde{u}_{j}\right|^{p}\right)\left|\widetilde{u}_{j}\right|^{p-2} \widetilde{u}_{j}\right) \varphi=0
\end{aligned}
$$

uniformly in $\varphi \in E_{\lambda, A}$ with $\|\varphi\|_{\lambda, A} \leq 1$.

Proof By direct computation, we easily obtain $\widetilde{u}_{j} \rightarrow u$ in $E_{\lambda, A}$. The local compactness of the Sobolev embedding implies that, for any $r \geq 0$, we have

$$
\begin{aligned}
& \lim _{j \rightarrow \infty} \sup \operatorname{Re} \int_{B_{r}}\left(f\left(x,\left|u_{n_{j}}\right|^{p}\right)\left|u_{n_{j}}\right|^{p-2} u_{n_{j}}\right. \\
& \left.\quad-f\left(x, u_{n_{j}}-\widetilde{u}_{j}\right)\left|u_{n_{j}}-\widetilde{u}_{j}\right|^{p-2}\left(u_{n_{j}}-\widetilde{u}_{j}\right)-f\left(x,\left|\widetilde{u}_{j}\right|^{p}\right)\left|\widetilde{u}_{j}\right|^{p-2} \widetilde{u}_{j}\right) \bar{\varphi}=0
\end{aligned}
$$

uniformly in $\|\varphi\|_{\lambda, A} \leq 1$. For any $\varepsilon>0$, there is $r_{\varepsilon} \geq 0$ such that

$$
\lim _{j \rightarrow \infty} \sup \int_{B_{j} \backslash B_{r}}\left|\widetilde{u}_{j}\right|^{s} \leq \int_{\mathbb{R}^{N} \backslash B_{r}}|u|^{s} \leq \varepsilon
$$

for all $r \geq r_{\varepsilon}$. By the assumptions and the Hölder inequality, we have

$$
\begin{aligned}
& \lim _{j \rightarrow \infty} \sup \operatorname{Re} \int_{\mathbb{R}^{N}}\left(f\left(x,\left|u_{n_{j}}\right|^{p}\right)\left|u_{n_{j}}\right|^{p-2} u_{n_{j}}\right. \\
& \left.-f\left(x, u_{n_{j}}-\widetilde{u}_{j}\right)\left|u_{n_{j}}-\widetilde{u}_{j}\right|^{p-2}\left(u_{n_{j}}-\widetilde{u}_{j}\right)-f\left(x,\left|\widetilde{u}_{j}\right|^{p}\right)\left|\widetilde{u}_{j}\right|^{p-2} \widetilde{u}_{j}\right) \varphi \\
& =\lim _{j \rightarrow \infty} \sup \operatorname{Re} \int_{B_{j} \backslash B_{r}}\left(f\left(x,\left|u_{n_{j}}\right|^{p}\right)\left|u_{n_{j}}\right|^{p-2} u_{n_{j}}\right. \\
& \left.-f\left(x, u_{n_{j}}-\widetilde{u}_{j}\right)\left|u_{n_{j}}-\widetilde{u}_{j}\right|^{p-2}\left(u_{n_{j}}-\widetilde{u}_{j}\right)-f\left(x,\left|\widetilde{u}_{j}\right|^{p}\right)\left|\widetilde{u}_{j}\right|^{p-2} \widetilde{u}_{j}\right) \varphi \\
& \leq c_{1} \lim _{j \rightarrow \infty} \sup \int_{B_{j} \backslash B_{r}}\left(\left|u_{n_{j}}\right|^{p-1}+\left|\tilde{u}_{j}\right|^{p-1}\right)|\varphi| \\
& +c_{2} \lim _{j \rightarrow \infty} \sup \int_{B_{j} \backslash B_{r}}\left(\left|u_{n_{j}}\right|^{\alpha-1}+\left|\tilde{u}_{j}\right|^{\alpha-1}\right)|\varphi| \\
& \leq c_{1} \lim _{j \rightarrow \infty} \sup \left(\left\|u_{n_{j}}\right\|_{L^{\frac{p}{p-1}\left(B_{j} \backslash B_{r}\right)}}+\left\|\tilde{u}_{j}\right\|_{L^{\frac{p}{p-1}\left(B_{j} \backslash B_{r}\right)}}\right)\|\varphi\|_{L_{p}\left(B_{j} \backslash B_{r}\right)} \\
& +c_{2} \lim _{j \rightarrow \infty} \sup \left(\left\|u_{n_{j}}\right\|_{L_{\alpha}\left(B_{j} \backslash B_{r}\right)}^{\alpha-1}+\left\|\widetilde{u}_{j}\right\|_{L_{\alpha}\left(B_{j} \backslash B_{r}\right)}^{\alpha-1}\right)\|\varphi\|_{L_{\alpha}\left(B_{j} \backslash B_{r}\right)} \\
& \leq c_{3} \varepsilon^{\frac{p-1}{p}}+c_{4} \varepsilon^{\frac{\alpha-1}{\alpha}} \text {. }
\end{aligned}
$$

This proof is completed. 
Lemma 3.4 One has along a subsequence

$$
I_{\lambda}\left(u_{n}-\widetilde{u}_{n}\right) \rightarrow c-I_{\lambda}(u)
$$

and

$$
\left.I_{\lambda}^{\prime}\left(u_{n}-\widetilde{u}_{n}\right) \rightarrow 0 \quad \text { in } E_{\lambda}^{-1} \text { (the dual space of } E_{\lambda}\right) .
$$

Proof Combining Lemma 2.1 of [30] and the arguments of [31], one has

$$
\begin{aligned}
I_{\lambda}\left(u_{n}\right. & \left.-\widetilde{u}_{n}\right) \\
= & I_{\lambda}\left(u_{n}\right)-I_{\lambda}\left(\widetilde{u}_{n}\right) \\
& +\frac{\lambda}{p^{*}} \int_{\mathbb{R}^{N}} K(x)\left(\left|u_{n}\right|^{p^{*}}-\left|u_{n}-\widetilde{u}_{n}\right|^{p^{*}}-\left|\widetilde{u}_{n}\right|^{p^{*}}\right) \\
& +\frac{\lambda}{p} \int_{\mathbb{R}^{N}}\left(F\left(x,\left|u_{n}\right|^{p}\right)-F\left(x,\left|u_{n}-\widetilde{u}_{n}\right|^{p}\right)-F\left(x,\left|\widetilde{u}_{n}\right|^{p}\right)\right)+o(1) .
\end{aligned}
$$

By the Brézis-Lieb lemma [32], we get

$$
\lim _{n \rightarrow \infty} \int_{\mathbb{R}^{N}} K(x)\left(\left|u_{n}\right|^{p^{*}}-\left|u_{n}-\widetilde{u}_{n}\right|^{p^{*}}-\left|\widetilde{u}_{n}\right|^{p^{*}}\right)=0
$$

and

$$
\lim _{n \rightarrow \infty} \int_{\mathbb{R}^{N}}\left(F\left(x,\left|u_{n}\right|^{p}\right)-F\left(x,\left|u_{n}-\widetilde{u}_{n}\right|^{p}\right)-F\left(x,\left|\widetilde{u}_{n}\right|^{p}\right)\right)=0 .
$$

We now observe that $I_{\lambda}\left(u_{n}\right) \rightarrow c$ and $I_{\lambda}\left(\widetilde{u}_{n}\right) \rightarrow I_{\lambda}(u)$, which gives

$$
I_{\lambda}\left(u_{n}-\widetilde{u}_{n}\right) \rightarrow c-I_{\lambda}(u) .
$$

Moreover, by direct computation, we get

$$
\begin{aligned}
I_{\lambda}^{\prime}\left(u_{n}-\widetilde{u}_{n}\right) \varphi= & I_{\lambda}^{\prime}\left(u_{n}\right) \varphi-I_{\lambda}^{\prime}\left(\widetilde{u}_{n}\right) \varphi \\
& +\lambda \operatorname{Re} \int_{\mathbb{R}^{N}} K(x)\left(\left|u_{n}\right|^{p^{*}-2} u_{n}-\left|u_{n}-\widetilde{u}_{n}\right|^{p^{*}-2}\left(u_{n}-\widetilde{u}_{n}\right)-\left|\widetilde{u}_{n}\right|^{p^{*}-2} \widetilde{u}_{n}\right) \varphi \\
& +\lambda \operatorname{Re} \int_{\mathbb{R}^{N}}\left(f\left(x,\left|u_{n}\right|^{p}\right)\left|u_{n}\right|^{p-2} u_{n}\right. \\
& \left.-f\left(x,\left|u_{n}-\widetilde{u}_{n}\right|^{p}\right)\left|u_{n}-\widetilde{u}_{n}\right|^{p-2}\left(u_{n}-\widetilde{u}_{n}\right)-f\left(x,\left|\widetilde{u}_{n}\right|^{p}\right)\left|\widetilde{u}_{n}\right|^{p-2} \widetilde{u}_{n}\right) \varphi+o(1) .
\end{aligned}
$$

It then follows from the standard arguments that

$$
\lim _{n \rightarrow \infty} \operatorname{Re} \int_{\mathbb{R}^{N}} K(x)\left(\left|u_{n}\right|^{p^{*}-2} u_{n}-\left|u_{n}-\widetilde{u}_{n}\right|^{p^{*}-2}\left(u_{n}-\widetilde{u}_{n}\right)-\left|\widetilde{u}_{n}\right|^{p^{*}-2} \widetilde{u}_{n}\right) \varphi=0
$$

uniformly in $\|\varphi\|_{\lambda, A} \leq 1$. Combining Lemma 3.3, we get $I_{\lambda}^{\prime}\left(u_{n}-\widetilde{u}_{n}\right) \rightarrow 0$. The proof is completed. 
Let $u_{n}^{1}=u_{n}-\tilde{u}_{n}$, then $u_{n}-u=u_{n}^{1}+\left(\tilde{u}_{n}-u\right)$. Therefore, $u_{n} \rightarrow u$ in $E_{\lambda, A}$ if and only if $u_{n}^{1} \rightarrow 0$ in $E_{\lambda, A}$.

Note that

$$
\begin{aligned}
& I_{\lambda}\left(u_{n}^{1}\right)-\frac{1}{p} I_{\lambda}^{\prime}\left(u_{n}^{1}\right) u_{n}^{1} \\
& \quad=\left(\frac{1}{p}-\frac{1}{p^{*}}\right) \lambda \int_{\mathbb{R}^{N}} K(x)\left|u_{n}^{1}\right|^{p^{*}}+\lambda \int_{\mathbb{R}^{N}} \frac{1}{p}\left(\left|u_{n}^{1}\right|^{p} f\left(x,\left|u_{n}^{1}\right|^{p}\right)-F\left(x,\left|u_{n}^{1}\right|^{p}\right)\right) \\
& \quad \geq \frac{\lambda}{N} \int_{\mathbb{R}^{N}} K(x)\left|u_{n}^{1}\right|^{p^{*}} \\
& \quad \geq \frac{\lambda}{N} K_{\min }\left\|u_{n}^{1}\right\|_{p^{*}}^{p^{*}}
\end{aligned}
$$

where $K_{\min }=\inf _{x \in \mathbb{R}^{N}} K(x)>0$. Together with Lemma 3.4, one has

$$
\left\|u_{n}^{1}\right\|_{p^{*}}^{p^{*}} \leq \frac{N\left(c-I_{\lambda}(u)\right)}{\lambda K_{\min }}+o(1) .
$$

In the following, we consider the energy level of the functional $I_{\lambda}$ below which the $(P S)_{c}$ condition holds.

Denote $V_{b}(x):=\max \{V(x), b\}$, where $b$ is the positive constant in assumption $\left(\mathrm{V}_{0}\right)$. Since the set $v_{b}$ has a finite measure, combining the fact that $u_{n}^{1} \rightarrow 0$ in $L_{\text {loc }}^{p}\left(\mathbb{R}^{N}\right)$, we get

$$
\int_{\mathbb{R}^{N}} V(x)\left|u_{n}^{1}\right|^{p}=\int_{\mathbb{R}^{N}} V_{b}(x)\left|u_{n}^{1}\right|^{p}+o(1) .
$$

Furthermore, by $\left(\mathrm{K}_{0}\right)$ and $\left(\mathrm{H}_{1}\right)-\left(\mathrm{H}_{3}\right)$, there exists $C_{b}>0$ such that

$$
\int_{\mathbb{R}^{N}}\left(K(x)\left|u_{n}^{1}\right|^{p^{*}}+\left|u_{n}^{1}\right|^{p} f\left(x,\left|u_{n}^{1}\right|^{p}\right)\right) \leq b\left\|u_{n}^{1}\right\|_{p}^{p}+C_{b}\left\|u_{n}^{1}\right\|_{p^{*}}^{p^{*}}
$$

Let $S$ be the best Sobolev constant of the immersion

$$
S\|u\|_{p^{*}}^{p} \leq \int_{\mathbb{R}^{N}}|\nabla u|^{p} \quad \text { for all } u \in W^{1, p}\left(\mathbb{R}^{N}\right) .
$$

Lemma 3.5 There exists $\alpha_{0}>0$ (independent of $\lambda$ ) such that, for any $(P S)_{c}$ sequence $\left\{u_{n}\right\} \subset$ $E_{\lambda, A}$ for $I_{\lambda}$ with $u_{n} \rightarrow u$, either $u_{n} \rightarrow u$ in $E_{\lambda, A}$ or $c-I_{\lambda}(u) \geq \alpha_{0} \lambda^{1-\frac{N}{p}}$.

Proof Arguing by contradiction, assume that $u_{n} \nrightarrow u$, then

$$
\lim \inf _{n \rightarrow \infty}\left\|u_{n}^{1}\right\|_{\lambda, A}>0
$$

Combining the Sobolev inequality, (3.2) and (3.3), we get

$$
\begin{aligned}
S\left\|u_{n}^{1}\right\|_{p^{*}}^{p} & \leq \int_{\mathbb{R}^{N}}\left|\nabla u_{n}^{1}\right|^{p} \\
& \leq \int_{\mathbb{R}^{N}}\left(\left|\nabla u_{n}^{1}+i \lambda^{\frac{1}{p}} A(x) u_{n}^{1}\right|^{p}+\lambda V(x)\left|u_{n}^{1}\right|^{p}\right)-\lambda \int_{\mathbb{R}^{N}} V(x)\left|u_{n}^{1}\right|^{p} \\
& =\lambda \int_{\mathbb{R}^{N}}\left(K(x)\left|u_{n}^{1}\right|^{p^{*}}+\left|u_{n}^{1}\right|^{p} f\left(x,\left|u_{n}^{1}\right|^{p}\right)\right)-\lambda \int_{\mathbb{R}^{N}} V_{b}(x)\left|u_{n}^{1}\right|^{p}+o(1)
\end{aligned}
$$




$$
\begin{aligned}
& \leq \lambda b\left\|u_{n}^{1}\right\|_{p}^{p}+\lambda C_{b}\left\|u_{n}^{1}\right\|_{p^{*}}^{p^{*}}-\lambda b\left\|u_{n}^{1}\right\|_{p}^{p}+o(1) \\
& =\lambda C_{b}\left\|u_{n}^{1}\right\|_{p^{*}}^{p^{*}}+o(1)
\end{aligned}
$$

which further gives

$$
\begin{aligned}
S & \leq \lambda C_{b}\left\|u_{n}^{1}\right\|_{p^{*}}^{p^{*}-p}+o(1) \\
& \leq \lambda C_{b}\left(\frac{N\left(c-I_{\lambda}(u)\right)}{\lambda K_{\min }}\right)^{\frac{p}{N}}+o(1) \\
& =\lambda^{1-\frac{p}{N}} C_{b}\left(\frac{N}{K_{\min }}\right)^{\frac{p}{N}}\left(c-I_{\lambda}(u)\right)^{\frac{p}{N}}+o(1) .
\end{aligned}
$$

Denote $\alpha_{0}=S^{\frac{N}{p}} C_{b}^{-\frac{N}{p}} N^{-1} K_{\min }$, then

$$
\alpha_{0} \lambda^{1-\frac{N}{p}} \leq c-I_{\lambda}(u)+o(1)
$$

We obtain the desired conclusion.

Lemma 3.6 There exists a constant $\alpha_{0}>0$ (independent of $\lambda$ ) such that if a $(P S)_{c}$ sequence $\left\{u_{n}\right\} \subset E_{\lambda, A}$ for $I_{\lambda}$ satisfies $c \leq \alpha_{0} \lambda^{1-\frac{N}{p}}$, the sequence $\left\{u_{n}\right\}$ has a strongly convergent subsequence in $E_{\lambda, A}$.

Proof By the fact that $I_{\lambda}(u) \geq 0$ and Lemma 3.5, we easily get the required conclusion.

Now, we consider $\lambda \geq 1$ and prove that the energy functional $I_{\lambda}$ possesses the mountain pass structure.

Lemma 3.7 Under the assumptions of Theorem 2, there exist $\alpha_{\lambda}, \rho_{\lambda}>0$ such that

$$
I_{\lambda}(u)>0 \quad \text { if } 0<\|u\|_{\lambda, A}<\rho_{\lambda} \quad \text { and } \quad I_{\lambda}(u) \geq \alpha_{\lambda} \quad \text { if }\|u\|_{\lambda, A}=\rho_{\lambda} .
$$

Proof The proof of Lemma 3.7 is similar to the one of Lemma 4.1 in [8].

Lemma 3.8 For any finite dimensional subspace $F \subset E_{\lambda, A}$, we have

$$
I_{\lambda}(u) \rightarrow-\infty, \quad u \in F \text { as }\|u\|_{\lambda, A} \rightarrow \infty
$$

Proof By assumptions $\left(\mathrm{K}_{0}\right)$ and $\left(\mathrm{H}_{3}\right)$, one has

$$
I_{\lambda}(u) \leq \frac{1}{p}\|u\|_{\lambda, A}^{p}-\lambda a_{0}\|u\|_{q}^{q} \quad \text { for all } u \in E_{\lambda, A} .
$$

Since all norms in a finite-dimensional space are equivalent, in connection with $q>p$, we obtain the desired conclusion.

For $\lambda$ large enough and $c_{\lambda}$ small sufficiently, $I_{\lambda}$ satisfies $(P S)_{c_{\lambda}}$ condition by Lemma 3.6. Furthermore, we will find special finite-dimensional subspace, by which we establish sufficiently small minimax levels. 
Define the functional

$$
\Phi_{\lambda}(u)=\frac{1}{p} \int_{\mathbb{R}^{N}}\left(\left|\nabla u+i \lambda^{\frac{1}{p}} A(x) u\right|^{p}+\lambda V(x)|u|^{p}\right)-\lambda a_{0} \int_{\mathbb{R}^{N}}|u|^{q} .
$$

It is easy to see that $\Phi_{\lambda} \in C^{1}\left(E_{\lambda, A}\right)$ and $I_{\lambda}(u) \leq \Phi_{\lambda}(u)$ for all $u \in E_{\lambda, A}$. Note that

$$
\inf \left\{\int_{\mathbb{R}^{N}}|\nabla \phi|^{p}: \phi \in C_{0}^{\infty}\left(\mathbb{R}^{N}, \mathbb{R}\right),\|\phi\|_{L^{q}\left(\mathbb{R}^{N}\right)}=1\right\}=0
$$

For any $\delta>0$, there is $\phi_{\delta} \in C_{0}^{\infty}\left(\mathbb{R}^{N}, \mathbb{R}\right)$ with $\left\|\phi_{\delta}\right\|_{L^{q}\left(\mathbb{R}^{N}\right)}=1$ and $\operatorname{supp} \phi_{\delta} \subset B_{r_{\delta}}(0)$ such that $\left\|\nabla \phi_{\delta}\right\|_{p}^{p}<\delta$. Let $e_{\lambda}(x)=\phi_{\delta}(\sqrt[p]{\lambda} x)$, then supp $e_{\lambda} \subset B_{\lambda^{-\frac{1}{p}}}(0)$. For any $t \geq 0$, we have

$$
\begin{aligned}
\Phi_{\lambda}\left(t e_{\lambda}\right) & =\frac{t^{p}}{p}\left\|e_{\lambda}\right\|_{\lambda, A}^{p}-a_{0} \lambda t^{q} \int_{\mathbb{R}^{N}}\left|\phi_{\delta}(\sqrt[p]{\lambda} x)\right|^{q} \\
& =\lambda^{1-\frac{N}{p}} J_{\lambda}\left(t \phi_{\delta}\right),
\end{aligned}
$$

where

$$
J_{\lambda}(u)=\frac{1}{p} \int_{\mathbb{R}^{N}}\left(\left|\nabla u+i \lambda^{\frac{1}{p}} A(x) u\right|^{p}+V\left(\lambda^{-\frac{1}{p}} x\right)|u|^{p}\right)-a_{0} \int_{\mathbb{R}^{N}}|u|^{q} .
$$

We derive that

$$
\max _{t \geq 0} J_{\lambda}\left(t \phi_{\delta}\right) \leq \frac{q-p}{p q\left(q a_{0}\right)^{\frac{p}{q-p}}}\left(\int_{\mathbb{R}^{N}}\left(\left|\nabla \phi_{\delta}\right|^{p}+\left(A\left(\lambda^{-\frac{1}{p}} x\right)+V\left(\lambda^{-\frac{1}{p}} x\right)\right)\left|\phi_{\delta}\right|^{p}\right)\right)^{\frac{q}{q-p}} .
$$

Observe that $A(0)=0, V(0)=0$ and $\left\|\nabla \phi_{\delta}\right\|_{p}^{p}<\delta$. Therefore, there exists $\Lambda_{\delta}>0$ such that for all $\lambda \geq \Lambda_{\delta}$, we have

$$
\max _{t \geq 0} I_{\lambda}\left(t \phi_{\delta}\right) \leq\left(\frac{q-p}{p q\left(q a_{0}\right)^{\frac{p}{q-p}}}(5 \delta)^{\frac{q}{q-p}}\right) \lambda^{1-\frac{N}{p}}
$$

Lemma 3.9 Under the assumptions of Theorem 2, for any $\sigma>0$, there is $\Lambda_{\sigma}>0$ such that for each $\lambda \geq \Lambda_{\sigma}$, there exists $\bar{e}_{\lambda} \in E_{\lambda, A}$ with $\left\|\bar{e}_{\lambda}\right\|_{\lambda, A}>\rho_{\lambda}, I_{\lambda}\left(\bar{e}_{\lambda}\right) \leq 0$ and

$$
\max _{t \geq 0} I_{\lambda}\left(t \bar{e}_{\lambda}\right) \leq \sigma \lambda^{1-\frac{N}{p}}
$$

where $\rho_{\lambda}$ is defined in Lemma 3.7.

Proof For any $\sigma>0$, we can choose $\delta<0$ so small that

$$
\frac{q-p}{p q\left(q a_{0}\right)^{\frac{p}{q-p}}}(5 \delta)^{\frac{q}{q-p}} \leq \sigma
$$

Denote $e_{\lambda}(x)=\phi_{\delta}(\sqrt[p]{\lambda} x)$ and $\Lambda_{\sigma}=\Lambda_{\delta}$. Let $\bar{t}_{\lambda}>0$ be such that $\bar{t}_{\lambda}\left\|e_{\lambda}\right\|_{\lambda, A}>\rho_{\lambda}$ and $I_{\lambda}\left(t e_{\lambda}\right) \leq 0$ for all $t \geq \bar{t}_{\lambda}$. Then, combining (3.4), $\bar{e}_{\lambda}=\bar{t}_{\lambda} e_{\lambda}$ meets the requirements. 


\section{Proof of Theorem 2}

In this section, we give the proof of Theorem 2.

Proof By Lemma 3.9, for any $\sigma>0$ with $0<\sigma<\alpha_{0}$, we choose $\Lambda_{\sigma}>0$ and define the minimax value

$$
c_{\lambda}=\inf _{\gamma \in \Gamma_{\lambda}} \max _{t \in[0,1]} I_{\lambda}(\gamma(t)) \quad \text { with } c_{\lambda} \leq \sigma \lambda^{1-\frac{N}{p}} \text { for each } \lambda \geq \Lambda_{\sigma}
$$

where $\Gamma_{\lambda}=\left\{\gamma \in C\left([0,1], E_{\lambda, A}\right): \gamma(0)=0, \gamma(1)=\bar{e}_{\lambda}\right\}$

Lemma 3.6 shows that $I_{\lambda}$ satisfies $(P S)_{c_{\lambda}}$ condition. Therefore, by the mountain pass theorem, there exists $u_{\lambda} \in E_{\lambda, A}$, which satisfies $I_{\lambda}\left(u_{\lambda}\right)=c_{\lambda}$ and $I_{\lambda}^{\prime}\left(u_{\lambda}\right)=0$. That is, $u_{\lambda}$ is a weak solution of (2.1). Furthermore, it is well known that $u_{\lambda}$ is the least energy solution of equation (2.1).

Moreover, together with $I_{\lambda}\left(u_{\lambda}\right) \leq \sigma \lambda^{1-\frac{N}{p}}$ and $I_{\lambda}^{\prime}\left(u_{\lambda}\right)=0$, we have

$$
\begin{aligned}
I_{\lambda}\left(u_{\lambda}\right)= & I_{\lambda}\left(u_{\lambda}\right)-\frac{1}{\theta} I_{\lambda}^{\prime}\left(u_{\lambda}\right)\left(u_{\lambda}\right) \\
= & \left(\frac{1}{p}-\frac{1}{\theta}\right)\left\|u_{\lambda}\right\|_{\lambda, A}^{p}+\left(\frac{1}{\theta}-\frac{1}{p^{*}}\right) \lambda \int_{\mathbb{R}^{N}} K(x)\left|u_{\lambda}\right|^{p^{*}} \\
& +\lambda \int_{\mathbb{R}^{N}}\left(\frac{1}{\theta}\left|u_{\lambda}\right|^{p} f\left(x,\left|u_{\lambda}\right|^{p}\right)-\frac{1}{p} F\left(x,\left|u_{\lambda}\right|^{p}\right)\right) \\
\geq & \left(\frac{1}{p}-\frac{1}{\theta}\right)\left\|u_{\lambda}\right\|_{\lambda, A}^{p} .
\end{aligned}
$$

By inequality (2.3), we obtain

$$
\frac{\theta-p}{p \theta} \int_{\mathbb{R}^{N}}\left(\left|\nabla u_{\lambda}\right|^{p}+\lambda V(x)\left|u_{\lambda}\right|^{p}\right) \leq \sigma \lambda^{1-\frac{N}{p}} .
$$

The proof is complete.

\section{Competing interests}

The authors declare that they have no competing interests.

\section{Authors' contributions}

The authors contributed equally in this article. They read and approved the final manuscript.

\section{Acknowledgements}

The authors would like to appreciate the referees for their precious comments and suggestions about the original manuscript. This research was supported by the National Natural Science Foundation of China (11271364) and the Fundamental Research Funds for the Central Universities (2012QNA46).

Received: 3 April 2013 Accepted: 28 August 2013 Published: 05 Nov 2013

References

1. Ambrosetti, A, Rabinowitz, PH: Dual variational methods in critical point theory and applications. J. Funct. Anal. 14, 349-381 (1973)

2. Bartsch, T, Dancer, E, Peng, S: On multi-bump semi-classical bound states of nonlinear Schrödinger equations with electromagnetic fields. Adv. Differ. Equ. 11, 781-812 (2006)

3. Benci, V: On critical point theory of indefinite functions in the presence of symmetries. Trans. Am. Math. Soc. 274 533-572 (1982)

4. Brézis, H, Nirenberg, L: Positive solutions of nonlinear elliptic equation involving critical Sobolev exponents. Commun. Pure Appl. Math. 16, 437-477 (1983) 
5. Cingolani, S: Semiclassical stationary states of nonlinear Schrödinger equation with an external magnetic field. J. Differ. Equ. 188, 52-79 (2003)

6. Clapp, M, Ding, YH: Minimal nodal solutions of a Schrödinger equation with critical nonlinearity and symmetric potential. Differ. Integral Equ. 16, 981-992 (2003)

7. Del Pino, M, Felmer, PL: Semi-classical states for nonlinear Schrödinger equations. J. Funct. Anal. 149, $245-265$ (1997)

8. Ding, YH, Lin, FH: Solutions of perturbed Schrödinger equations with critical nonlinearity. Calc. Var. 30, 231-249 (2007)

9. Ding, YH, Szulkin, A: Bound states for semilinear Schrödinger equations with sign-changing potential. Calc. Var. Partial Differ. Equ. 29, 397-419 (2007)

10. Esteban, M, Lions, PL: Stationary solutions of nonlinear Schrödinger equation with an external magnetic field. In: PDE and Calculus of Variations: In Honor of E De Giorgi, pp. 369-408. Birkhäuser, Basel (1990)

11. Floer, A, Weinstein, A: Nonspreading wave packets for the cubic Schrödinger equation with a bounded potential. J. Funct. Anal. 69, 397-408 (1986)

12. Grossi, M: Some results on a class of nonlinear Schrödinger equations. Math. Z. 235, 687-705 (2000)

13. Jeanjean, L, Tanaka, K: Singularly perturbed elliptic problems with superlinear or asymptotically linear nonlinearities. Calc. Var. Partial Differ. Equ. 21, 287-318 (2004)

14. Kang, X, Wei, J: On interacting bumps of semi-classical states of nonlinear Schrödinger equations. Adv. Differ. Equ. 5 899-928 (2000)

15. Li, YY: On a singularly perturbed elliptic equation. Adv. Differ. Equ. 2, 955-980 (1997)

16. Oh, YG: Existence of semiclassical bound states of nonlinear Schrödinger equations with potentials of the class $(V)_{a}$. Commun. Partial Differ. Equ. 13, 1499-1519 (1988)

17. Oh, YG: On positive multi-lump bound states of nonlinear Schrödinger equations under multiple well potential Commun. Math. Phys. 131, 223-253 (1990)

18. Pistoia, A: Multi-peak solutions for a class of some results on a class of nonlinear Schrödinger equations. Nonlinear Differ. Equ. Appl. 9, 69-91 (2002)

19. Tang, Z: On the least energy solutions of nonlinear Schrödinger equations with electromagnetic fields. Comput. Math. Appl. 54, 627-637 (2007)

20. Tang, Z: Multi-bump bound states of nonlinear Schrödinger equations with electromagnetic fields and critical frequency. J. Differ. Equ. 245, 2723-2748 (2008)

21. Wang, F: On an electromagnetic Schrödinger equation with critical growth. Nonlinear Anal. 69, 4088-4098 (2008)

22. Alves, CO, Ding, YH: Multiplicity of positive solutions to a $p$-Laplacian equation involving critical nonlinearity. J. Math. Anal. Appl. 279, 508-521 (2003)

23. El Khalil, A, El Manouni, S, Ouanan, M: On some nonlinear elliptic problems for $p$-Laplacian in $\mathbb{R}^{N}$. NoDEA Nonlinear Differ. Equ. Appl. 15, 295-307 (2008)

24. Fan, XL: $p(x)$-Laplacian equations in $\mathbb{R}^{N}$ with periodic data and nonperiodic perturbations. J. Math. Anal. Appl. 341, 103-119 (2008)

25. Habib, SE, Tsouli, N: On the spectrum of the $p$-Laplacian operator for Neumann eigenvalue problems with weights. Electron. J. Differ. Equ. Conf. 14, 181-190 (2005)

26. Lê, A: Eigenvalue problems for the $p$-Laplacian. Nonlinear Anal. 64, 1057-1099 (2006)

27. Liu, CG, Zheng, YQ: Existence of nontrivial solutions for $p$-Laplacian equations in $\mathbb{R}^{N}$. J. Math. Anal. Appl. 380, 669-679 (2011)

28. Manásevich, R, Mawhin, J: Boundary value problems for nonlinear perturbations of vector $p$-Laplacian-like operators. J. Korean Math. Soc. 5, 665-685 (2000)

29. Mawhin, J, Willem, M: Critical Point Theory and Hamiltonian Systems. Springer, New York (1989)

30. Li, YY, Guo, QQ, Niu, PC: Global compactness results for quasilinear elliptic problems with combined critical Sobolev-Hardy terms. Nonlinear Anal. 74, 1445-1464 (2011)

31. Ghoussoub, N, Yuan, C: Multiple solutions for quasi-linear PDES involving the critical Sobolev and Hardy exponents Trans. Am. Math. Soc. 352, 5703-5743 (2000)

32. Brézis, H, Lieb, E: A relation between pointwise convergence of functions and convergence of functional. Proc. Am. Math. Soc. 88, 486-490 (1983)

10.1186/1687-2770-2013-216

Cite this article as: Zhang and Jiang: Solutions of perturbed $p$-Laplacian equation with critical nonlinearity and magnetic fields. Boundary Value Problems 2013, 2013:216

\section{Submit your manuscript to a SpringerOpen ${ }^{\ominus}$ journal and benefit from:}

$\checkmark$ Convenient online submission

Rigorous peer review

- Immediate publication on acceptance

- Open access: articles freely available online

- High visibility within the field

- Retaining the copyright to your article

Submit your next manuscript at $>$ springeropen.com 